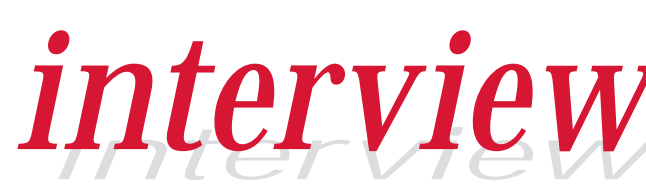

\title{
Genomics and drugs
}

\author{
An interview with Peter N. Goodfellow, Senior VicePresident of Discovery Research, GlaxoSmithKline
}

EMBO reports (ER): M any people talk about the impact that genomics and proteomics could have on biomedical research and healthcare. Do you see this as the next revolution in medical research?

Peter G oodfellow (PG): Well, I think that biomedical research has already gone through an absolute revolution over the past 50 years. Let me give you an example: in my first paper, I mapped one human gene to a human chromosome, and that was an article in Nature. O ne of the last papers I published concerned the use of radiation hybrids that we made for chromosomal localization-we mapped 20,000 cDNAs in that paper. So there's quite a revolution that's already taken place over the span of my scientific career.

\section{"Genomics might improveyour success rate because you might be able to answer some questions about specificity, but it's not going to make drug discovery a lot quicker."}

ER: So when will the advances in genomics and proteomics translate into new drugs?

PG: Experience tells us that it will take a long time. Let me give you an example: the first experiment I did when I went to the Imperial Cancer Research Fund [in London, UK] was to make a monoclonal antibody to the EG F receptor because the guy in the lab next door wandered in and asked for my help. I had no idea what the EGF receptor was, but I came up with a trick to make the antibody. Mike Waterfield used the antibody to purify the EG $F$ receptor, got enough amino-terminal sequence to show that it was related to Erb-B and that was the first

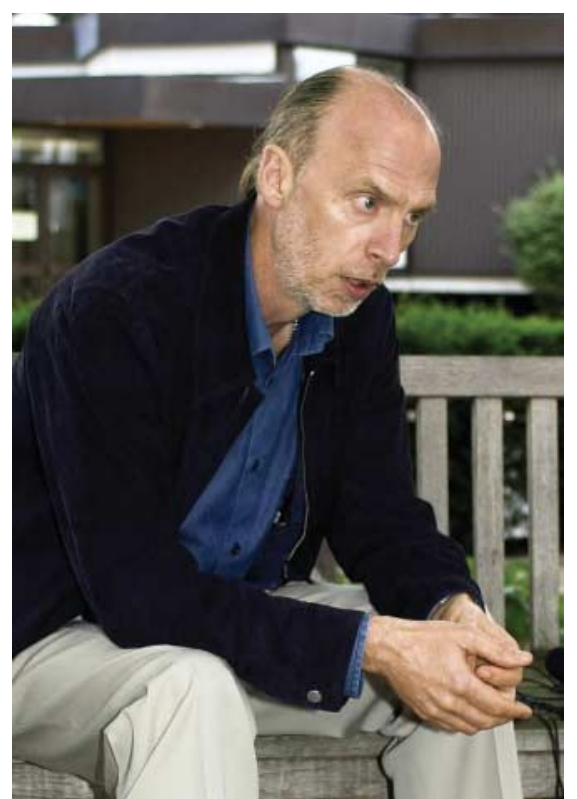

link between the EGF receptor and cancer. The first drug for targeting the EGF receptor family, Herceptin $₫$, came out 20 years later. So, from basic observation to treatment took 20 years. If you do that analysis for the drugs on the market today, the average time is about 17 years. There's a big lag time and there's a real danger that society will say, "We're just seeing the monoclonal antibodies now, but that's technology from the late '70s, early ' 80 s. W hen are we going to see the drugs from sequencing the human genome?" It's going to take 15 years before you see any of that. You have to explain the difficulties to people and make clear that it is a gradual change.

ER: Do you think this lag time could become a real danger for biomedical research?

PG: One of the reasons why I became involved in the pharmaceutical industry was the belief that it would be possible to use this information to make drugs. That was a very naive belief because I didn't know anything about making drugs. But if we can't use this information to make drugs, society's eventually going to say, "W hy are we spending all this money? Why should we give the $\mathrm{N}$ ational Institutes of Health (NIH) US\$20 billion a year? Where are all the new treatments?" Over the past decade or so, we've actually had relatively small advances in treatments. There are 1.2 million people who are diagnosed with cancer in America each year; 600,000 of those have metastatic disease, and 600,000 have localized disease. N ow if you look at those 600,000 who have metastatic disease when they're first diagnosed, 530,000 of them die from the disease. And if you look at those who have local disease when they're first diagnosed, 570,000 of those are cured. Which is another way of saying that we don't have treatments for cancer. And if we don't start delivering effective treatments, I think society will say to us, "Why should we bother to fund you?" M y own view is that we have to translate those advances into medicine as society has given us the money for basic research because they believe that it will be translated into medicine.

\section{"The only way that I can think of is if we could make a drug for each target before we knew we were interested in the target."}

ER: Do you think that genomics might help to speed up the process of drug development and make it more efficient?

PG: No. The sequence of the human genome has given us all the human genes, 


\section{science $\&$ society}

which means that the problem of specificity is solved in theory. So, if you make a drug against a kinase, you can ask the question, "D oes this drug inhibit just the kinase I'm interested in, or does it inhibit a whole load of other kinases as well?" But now that we have the human genome, when you are interested in a particular molecule, you can at least ask whether it interacts with any other potential targets. Genomics might improve your success rate because you might be able to answer some questions about specificity, but it's not going to make drug discovery a lot quicker. Genetics, theoretically, could give you a better definition of disease, and then it's possible that you could target your therapies better. If you can target your therapies better, it may be possible to start reducing development times. I say 'may' because it takes a small molecule six years to go from target through to the clinic-if everything works well-and then in the clinic it takes another seven years to go from its first trial in humans to the market. If you could target the population better you could do smaller trials, which theoretically would go through faster, but you're not really going to be able to reduce the overall time.

The only way that I can think of is if we could make a drug for each target before we knew we were interested in the target. This would take five years out of the process right up front. This is an experiment that we're doing with nuclear receptors. We've actually made drugs to the majority of these receptors, so you can reverse the question and say, "We've got a drug, now let's go find a disease." We could probably make a drug against every kinase today if we had the willpower and the funding. And then you change the problem, because you just say, "It's on the shelf. W here's the disease?"

\section{"There's a reason why we have regulation and that reason is thalidomide."}

ER:You'd still have to go through the clinical phase.

PG: Yes, but as I've said, it would take five years off the overall process. So that's one approach. I'm not sure how you could seriously reduce the time of the clinical component. It's about testing for safety and efficacy.

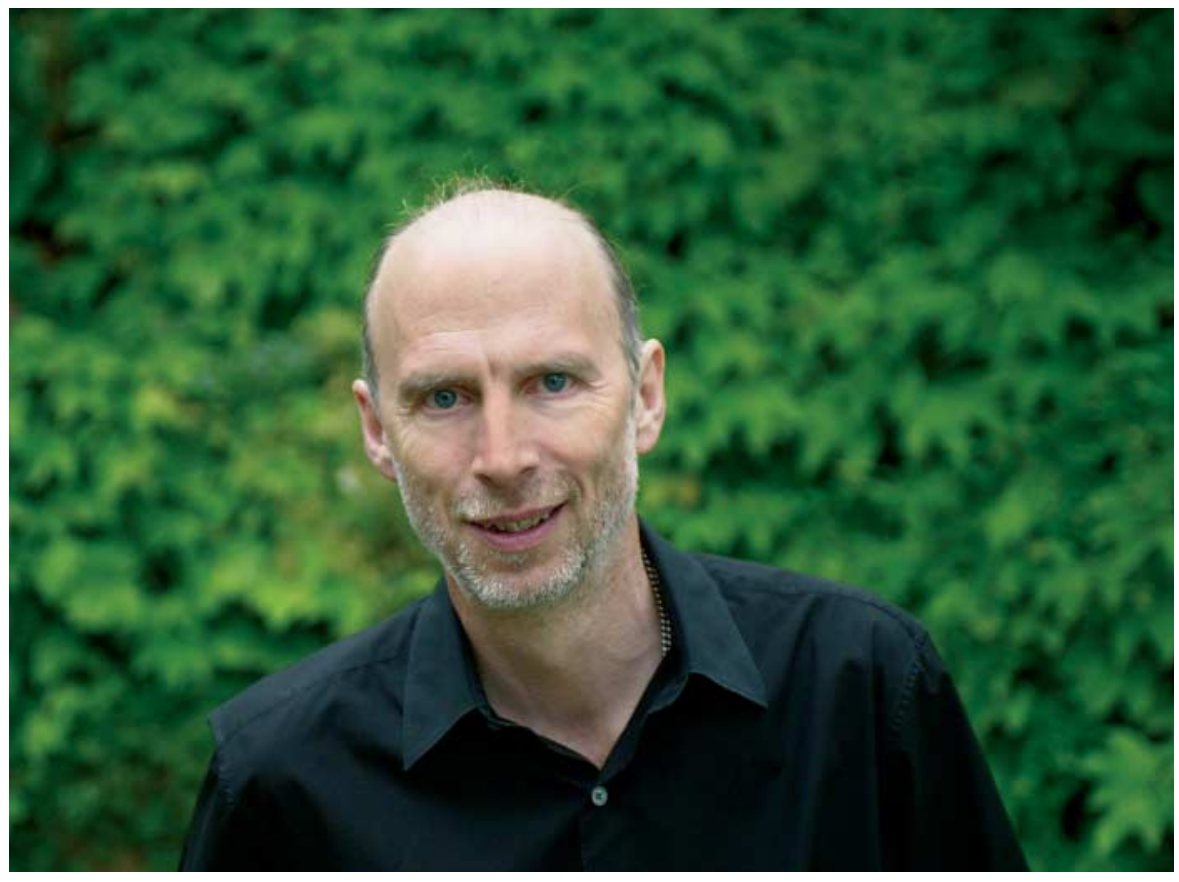

If you've enriched the population who will benefit from the drug, then all you need is sufficient time to see the efficacy signal. But would you be happy with just the efficacy signal? I can prove to you that this drug works over a period of one week but what happens if you take the drug for two years? Getting around safety is a problem. There is one solution: if you had surrogates that society or regulators were happy with then you wouldn't have to do such extensive trials to show outcomes. This is a real problem in cardiovascular disease; it could be a real problem in Alzheimer's disease. We were able to debate and agree that lowering cholesterol was probably a good thing, so we lowered cholesterol before we could actually prove that it was a good idea. The epidemiology said it must be true, but we didn't actually know that people who had high levels of cholesterol and then reduced those levels would actually reduce their risk of heart disease. But the statins got on the market and then eventually the outcome data was collected. That is one of the few surrogates that the regulatory authorities will accept.

ER: Do you think the US Food and Drug Administration (FDA) and European Medicines Evaluation Agency (EMEA) are becoming more stringent when approving drugs? Approvals by both agencies have dropped, and some say that even penicillin would not be approved today for safety reasons.
PG : I think it's true that you would have to work much harder today to get penicillin approved. And I think that is good. There's a reason why we have regulation and that reason is thalidomide. Regulation is a social construct. It's what we decide to do in order to get drugs. When it came to HIV and AIDS, the AIDS activists, the industry and the regulators actually changed the rules. We decided that it was acceptable if a drug only reduced viral load-that was good enough. So, we can construct our regulation according to what society actually wants. At the moment we're focused on safety and that is a good thing.

ER: How flexible are the FDA and the EMEA about changing the rules for regulating specific drugs, such as antibiotics, that are of particular interest?

PG: I would say that the FDA and the EMEA are social constructs, so we can decide as a society what level of risk we're happy with. We could reduce the time it takes for development if we had conditional approval, which is what they did with HIV drugs. You can sell the drug and recoup some of the costs that you've expended, but for the first two years you can only sell it to 10,000 patients or 100,000 patients, or you have to have more stringent conditions for it being on the market. So we can construct other ways of regulation. 
ER: 0 ther promises being made include better prevention of disease in people with a certain genetic make-up.

PG: Essentially, you know that you can prevent lung cancer and heart disease and have an enormous social impact by actually preventing people from smoking. And we've signally failed to do that in our society. So prevention just turns out to be a horrible problem for us, and if you had prognostic tests, I'm not sure how good that's going to be, to be quite honest. You could argue maybe that cholesterol testing has caused a fall in cardiovascular disease in the past decade. But if we really wanted to do something with diagnostic technology in terms of genetics, it would actually mostly be prognostic, and then you need to do something preventive, otherwise why bother? And that I think is a big problem. On the other hand, if genetics can give you a better definition of disease, then it will potentially give you benefits in the short term. So if you can determine that all schizophrenics are not actually all suffering from schizophrenia, but there are five different diseases responsible that respond differentially to existing therapies, this could make an enormous difference. The recent study on Iressa ${ }^{\circledR}$ is a good example, where they showed that patients who had a mutation in the EGF receptor were responding better. So that's a good example where somatic genetics can give you much better targeting of drugs. This would've made the trials smaller and it would've made everything work much better if they'd known that before they started the trial.

\section{"But if we really wanted to do something with diagnostic technology in terms of genetics, it would actually mostly be prognostic, and then you need to do something preventive, otherwise why bother?"}

ER: Coming back to the example you made earlier about the survival chances of people with metastatic versus localized cancercan diagnosis be improved?

PG : We focus on molecular medicine, but if I had a wish list, it would probably be for imaging because imaging can unlock all sorts of things like cardiovascular disease. If we can develop ultrasound to look for plaque formation, which is just on the cusp of what we can do at the moment, that would transform how you did the trial, as long as the regulators would accept reduction in plaque sizes being a surrogate for outcome. And of course with cancer and early diagnosis of cancer, if you could push those with metastatic cancer into the local disease group, that would have a big impact. But that's not molecular medicine, that's modern medicine.

\section{"We focus on molecular medicine but if I had a wish list, it would probably befor imaging because imaging can unlock all sorts of things..."}

ER: What big challenges do you see for biomedical research, disease-wise?

PG: I personally believe the biggest challenge is to make a vaccine for HIV. If I had a single wish, I would say a vaccine for HIV because I believe that what has happened in sub-Saharan Africa could happen in other parts of the world. My second wish would probably be a vaccine for malaria, and my third wish would be a vaccine for tuberculosis [TB]. And then we can start working on the diseases that afflict our [W estern] society.

ER: But the pharmaceutical industry is not necessarily putting a lot of money into malaria and TB vaccine research.

PG: We actually have programmes in malaria and $T B$, and we work in partnership with the $\mathrm{NIH}$, Medicine Malaria Venture, Global Alliance on TB and many others. What is needed is a public-private partnership approach to drug discovery and development in diseases of the developing world where no commercially viable market exists. Companies provide technology in which they have invested for decades and their discovery, development and distribution expertise to the partnership. The public sector partners help fund the development costs, while also ensuring that the medicines and vaccines get to the people who need them.

ER: $O \mathrm{n}$ the other hand, you have real blockbusters that are taken by hundreds of millions of people.
PG : Sure, that's how the system works. It's also true that we spend, as an industry, twice as much as the next high-tech industry on $R \& D$. So we spend, as a percentage of sales, between 15 and $20 \%$. Telecoms are down at about $7 \%$. We can change the rules, which Europe has basically done by giving us less money for our drugs and by regulating how much money we can make. Well, that will just get translated back into the margin, which gets translated back into the amount of $R \& D$ invested. And we'll see a decrease in investment in $R \& D$ and we'll also see pharmaceutical companies migrating to America, which is effectively what's been happening over the past decade.

ER: Do you think pharmaceutical companies will then start concentrating on lifestyle drugs and products that are guaranteed money-makers rather than disease drugs?

PG: Well, what do you mean by lifestyle? Because if you're suffering from erectile dysfunction, then do you regard that as a lifestyle issue or do you regard that as a medical issue? If you're suffering from depression, is that a lifestyle issue or is that a medical issue? If you're suffering from obesity, is that a lifestyle issue or is that a medical issue? There's a clear group on one side that is a medical issue, and then there's a gradation all the way through to where you have something else, such as a person who has normal weight, is perfectly fit and healthy but who wants to lose weight. That may be still a medical condition but it's not about obesity per se. So that's one strand. The second strand is that the majority of people who work in the pharmaceutical industry actually want to make drugs to treat people with serious diseases-they actually want to do research that benefits mankind. If the CEO of the company came in and said, "We want to make drugs that just make people feel better about themselves," it would be very hard to motivate the workforce. So I don't see it in those terms. I think the real answer to your question is that people will pay for cures for

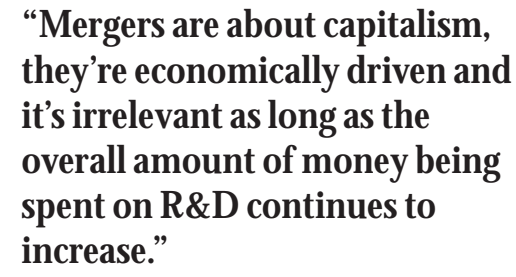


serious diseases. The financial drivers within capitalism mean that if your partner had cancer and it required you to sell your car in order to cure your partner, you wouldn't think twice about it. It's as simple as that.

ER: The Sanofi-Aventis partnership in Europe is the latest example of pharmaceutical company mergers. Does this really benefit drug development?

PG: I think what you have to look at is the overall social investment in $R \& D$ in drug discovery, and you won't find any evidence that this is decreasing. Mergers are about capitalism, they're economically driven and it's irrelevant as long as the overall amount of money being spent on $R \& D$ continues to increase. I personally don't believe there's a problem unless you think that large companies are less efficient at making drugs than small companies. You can compare the return on investment in biotech and the return on investment in major pharma and there's absolutely no difference. The truth is, making drugs is so difficult that you cannot predict success-what you find is wild success followed by wild failure, and that will lead to consolidation and companies merging. And this particular industry is still very unconsolidated compared with, for example, the car industry. The biggest automobile company in the world, General Motors, has about $40 \%$ market share. Now compare that with Pfizer, who have less than $10 \%$ market share. Again, the important thing is the overall investment in $R \& D$.

ER: Are larger pharmaceutical companies relying more on smaller drug discovery companies than they used to?

PG: As an industry-wide statement, I don't think there's been any change in the overall reliance on biotech companies or small companies. The truth is, it's so hard to get a drug, if you can get it, you'll get it from anywhere. I don't care where it comes from, and I don't care if somebody else does it rather than me, because it's so hard to get them.

\section{"The truth is, it's so hard to get a drug, if you can get it, you'll get it from anywhere. I don't care where it comes from, and I don't care if somebody else does it rather than me, because it's so hard to get them."}

ER: Do you think that this could improve in the next couple of years?

PG: At GSK, I've spent the past three years testing a hypothesis that if we generate very large data sets then we'll be able to extract from those data sets the knowledge that we need to make the process more predictable. Last year we generated over 100 million data points, and we are trying to work out ways to feed this information back into the process so that we can actually make it smarter. We start with a million compounds-small molecules that are starting points for drugs. And the thought experiment is, can we make each of those million compounds a drug before we screen them? The way you do that is to learn as much as you can about biology and chemistry and feed that information into those compounds. You throw out the compounds that will be toxic, you throw out the ones that will be insoluble, you throw out the ones that won't have the properties you want and only pursue those with properties you like. And every time we produce a new compound we're interested in, we test it in all the assays we have running against 500 different targets. By generating this data we make predictors that we feed back into that library. GSK has invested $f 260$ million in the past three years to automate a lot of the processes in expression, assay development, high-throughput screening and automated chemistry, to see if we can have leverage on scale. You can do this only if you're a large company. If you ask me if it is possible for a couple of guys in a garage to come up with a drug, I would say "sure". I'd be happy to go buy that drug and we'll develop it. But one day we're going to have to crack the central problem-that we still need to learn a lot more about compound selection-and when we find the solution, we'll have the drugs al ready sitting on the shelf.

ER: You said you went into the pharmaceutical industry because you wanted to apply biology to drug development. Have you ever regretted leaving academia?

PG : There are three things I miss from academia, in no particular order. I miss young people. When you're running a lab, which I did for most of my scientific career, you have graduate students and postdocs, and they always stay the same age. They come in and they go, and you don't realize you're getting older. And that was a big change when I went to industry because basically a large company is a hierarchy and so I don't talk to people under the age of 40 . I talk to people who talk to people who talk to people under the age of 40 . I also miss the crazy experimenting, where we'd sit around and think, "If DNA wasn't really hereditary material then it would mean X" and then you'd do a crazy experiment to test that. But now I'm interested in making drugs and so the playfulness disappears a little bit, and I miss that. The last thing I miss is when I was an academic I could go to any city in the world where there was a university and I would know somebody at that university or would know somebody who knew somebody at that university. And a lot of my students and postdocs would have similar sorts of branching networks-it's a bit like monks in medieval times. If you belonged to a particular order you could travel the world as part of that order, but I don't belong to that order anymore. I now belong to a different tribe, and I miss the tribe that I used to belong to. But the upside is that if you really want to translate some of the things that we created in academic research into things that do good for people then you have to do that in industry.

ER: Dr Goodfellow, thank you for the interview.

\section{Theinterview wasconducted by Holger Breithaupt and CarolineH adley.}

doi:10.1038/sj.embor.7400237 